In D. B. Kaber and G. A. Boy (Eds.), 'Advances in Cognitive Ergonomics', the Proceedings of the Third International Conference on Applied Human Factors and

Ergonomics, AHFE 2010 (pp. 450-459). Miami, Florida, USA, 17-20 July 2010:

CRC Press. doi.org/10.1201/EBK1439834916-c46

\title{
Using Mobile Information Sources to Increase Productivity and Quality
}

Peter Thorvald, Anna Brolin, Dan Högberg \& Keith Case

School of Technology and Society

University of Skövde

Skövde, Sweden

Mechanical and Manufacturing Engineering

Loughborough University

Loughborough, Leicestershire, UK

\begin{abstract}
This paper presents an experimental study made on the use of different kinds of information sources in manual assembly. The general idea is that only the necessary information should be presented to the worker and it should be presented where and when the worker needs it as this is believed to both save time and unload cognitive strain. To account for the latter two aspects of this thought, where and when, this paper investigates the use of a handheld unit as an information source in manual assembly. Having a mobile information system, such as a Personal Digital Assistant (PDA), that can be carried with you at all times, as opposed to a stationary one, such as a computer terminal, is hypothesized to greatly improve productivity and quality. Experimental results show that the use of a PDA significantly improves quality whereas productivity does not significantly improve.
\end{abstract}

Keywords: Assembly, information, range of information, PDA, information 
presentation

\section{INTRODUCTION}

Cost, environmental impact and quality are only a few of the challenges that automotive manufacturers need to face in order for their product to stand out against the competition. As a result of this increased competition in the industry, the demands on each individual worker become even greater. Manual assembly workers are given additional and better tools, more tasks, more quality checkpoints and less time to do all this in. Regarding information presentation, there seems to be a huge misconception within many of these organizations that more information leads to better quality. However, this is a belief that can be heavily criticized, especially since studies have shown that many manufacturers seem to have a problem getting their workers to attend to the information in the first place (Bäckstrand, et al., 2005, Thorvald, et al., 2008a, Thorvald, et al., 2008b). Adding even more information in such cases, which at first might seem like a sensible solution, would probably only lead to the workers paying less attention to information or reduced ability to sort out the relevant information. The likely outcome is that they 'will not see the forest for all trees'.

Looking at assembly domains, one can clearly see that information presentation technology is rarely adapted to humans. It's rather the other way around; the workers have to adapt their work strategies to fit the information technology. A clear example is the use of stationary computer terminals as information sources. These are not very mobile, as at best they can be turned or tilted to afford better visibility but can rarely be moved from their position. While working in a work cell which has limited space this might not be much of a problem. The information is never farther away than can be accessed by a turn of the head. However, in automotive assembly, which is where this paper sets out from, work cells can range in length from 1 to 15 meters with this paper focusing on truck chassis assembly which are typically about 11 meters. With this length of the work cell, you would also need a reasonable range of the information. A stationary computer terminal or even a large binder of printed papers, which can be very unpractical to move around and is common practice in many assembly factories, has limited range and depending on font and screen sizes can be difficult to see from a few meters away. When working in such a work cell, the worker has two choices for information gathering:

1. Use precious time and physical effort to move towards the information source and gather information.

2. Rely heavily on memory and experience to make correct choices in assembly.

Obviously, neither of these is desirable. Making the first choice would probably be considered the preferred alternative from an organizational perspective as we it would be supposed that the correct assembly is made as long as the assembly 
instructions are followed. However, assembly workers are often stressed and short of time and this behaviour would eventually increase stress and ultimately result in quality risks. The second alternative is better in terms of work load and stress but would obviously entail a huge quality risk if memory and experience fails.

So, how can this problem be remedied? Aside from the possibility of removing the need for information, logically there is only one way to go about this problem; move the information spatially closer to the task area. However, there are often many tasks spread out on for example a truck chassis, and it would be difficult to have separate information sources for all tasks. Instead, we suggest the use of a mobile information source such as a Personal Digital Assistant (PDA) which the worker can carry with him at all times. This would allow the worker quick and effortless access to information where and when it is needed.

\section{HYPOTHESIS}

The hypothesis for the experiment is as follows:

- Having a mobile information system, such as a PDA that can be carried at all times, as opposed to a stationary one, such as a computer terminal, improves productivity and quality.

The time saved due to the reduction of movement at the workstation is argued to benefit productivity in terms of hours per vehicle or similar measures. This decrease in physical strain ultimately leads to benefits in physical ergonomics and productivity. It is also plausible that the time to come to a decision of whether or not to consult the assembly instructions is diminished, as the internal calculation of the costs and benefits of the decision are more distinct.

The argument for better quality in the hypothesis is based on a cost-benefit (expectancy-value) reasoning (Jonides \& Mack, 1984, Wigfield \& Eccles, 2000) where it is believed that by reducing the cost of gathering the information, through walking distance, cognitive effort etc., subjects are more prone to use the information system than if it had been stationary and far away. However, the challenge is to determine what the actual effects on quality are.

\section{METHOD}

The challenge of satisfying testing of the productivity part of the hypothesis mainly consists of having a task with certain spatial properties. To be able to measure productivity the work cell needs to have a length of about 6 to 8 meters. This is simply to ensure that there is a considerable cost to return to the stationary information conveyer and this also corresponds well with the assembly tasks to be 
emulated. The second part though, quality, needs to be approached more delicately. It is believed that, because of the cognitive and physical strain of gathering information, assembly workers do not always judge it as worthwhile to do. However, this mentality might not occur until the worker is fairly experienced in assembly. Arguably, novices are unsure and tend not to stray from the assembly instructions too much. However, more experienced workers are more confident in their work and can sometimes value their own expertise too highly. Although it is plausible that the experience-factor is not necessary for this quality risk to occur, the situation might also arise where the worker has gathered the necessary information but forgotten it due to a prolonged stimulus-response gap (Dix, et al., 1998). In this case it could possibly be argued that the worker, instead of going back to the information source, puts more faith in their memory and thus might make an error. If a mobile information system is available at this point, the worker is argued to be less prone to trust their memory and consult the information system due to the low cost in cognitive and physical strain of gathering the information. This argument is made with a basis in cost-benefit and expectancy value theories (Jonides \& Mack, 1984, Wigfield \& Eccles, 2000).

The subjects were divided into two independent groups where each contained 12 to 14 university students/staff. Assembly workers, as experience and observations tells us, are a very heterogeneous group consisting of people of all ages, genders and personalities. In Skövde, Sweden, it is very common that young people spend a year or two in the automotive industry before moving on to University studies. Therefore, no extensive selection of subjects is undergone. This is to secure a mix of subjects that corresponds to the actual people working in assembly.

The task consisted of assembling several building blocks on a wooden chassis about 6 meters long. See figure 1 for a description of the laboratory layout. The test consisted of completing 20 iterations of the main task. Each task consisted of five subtasks where five different blocks were assembled with different bolts. As can be seen in figure 1 , the wooden structure, which is supposed to emulate a truck chassis, has two sides. Each main task was performed on one side and as the worker has come full circle around the chassis, she has performed two main tasks, each consisting of the assembly of five blocks to the chassis.

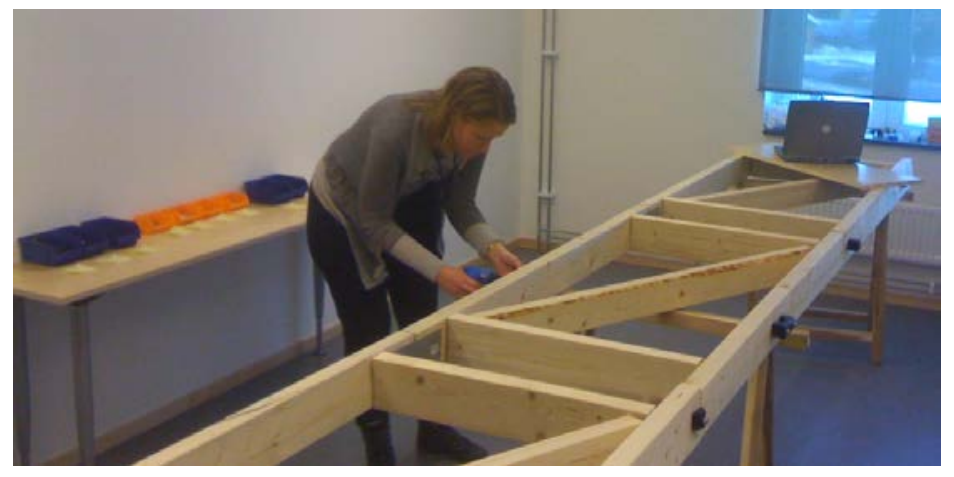


Figure 1. Picture of the work cell. Blocks assembled in the previous task can be seen in the foreground and a laptop as information source in the background.

In the two conditions that are used, one for each independent group, the independent variable is the information conveyer, i.e the stationary information source, a computer laptop (figure 1) and the handheld device which in this case is an iPod Touch. PDA setup is exactly the same as in figure 1, except that the laptop is removed and the worker is fitted with a PDA on their arm. Figure 2 shows a subject fitted and working with the PDA. In figure 2, it can be seen how the assembly worker, while mounting a block, is already focusing on what the next task is. His eyes are already fixated on the PDA.

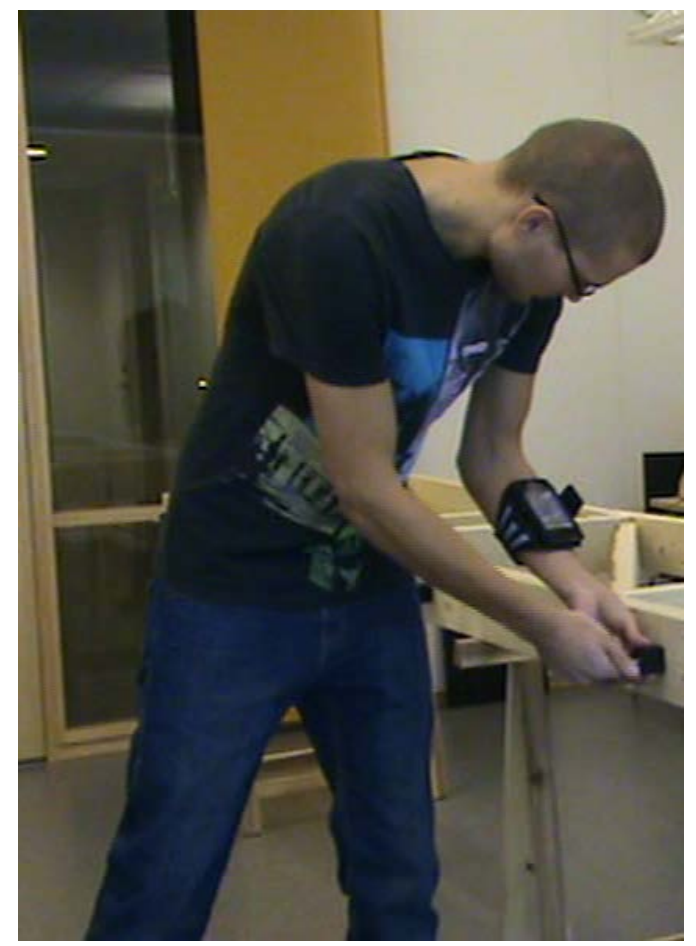

Figure 2. Example of an actual assembly worker interacting with the mobile device. The PDA is fastened on the subject's arm with a retail holster.

As the test continued the assembly information changed, blocks and bolts were assembled at different places or are replaced by other types of blocks and bolts. Both blocks and bolts were identified through unique four digit article numbers. Handheld power drivers were used to fasten the blocks to the chassis. 
The interface for the information conveyers was a newly developed prototype, designed for use in the automotive assembly industry, which is a domain that this test is intended to emulate in terms of the task and laboratory layout. See figure 3 for a screenshot from the software as shown on the PDA. The software looks identical on a computer terminal as it is web based.

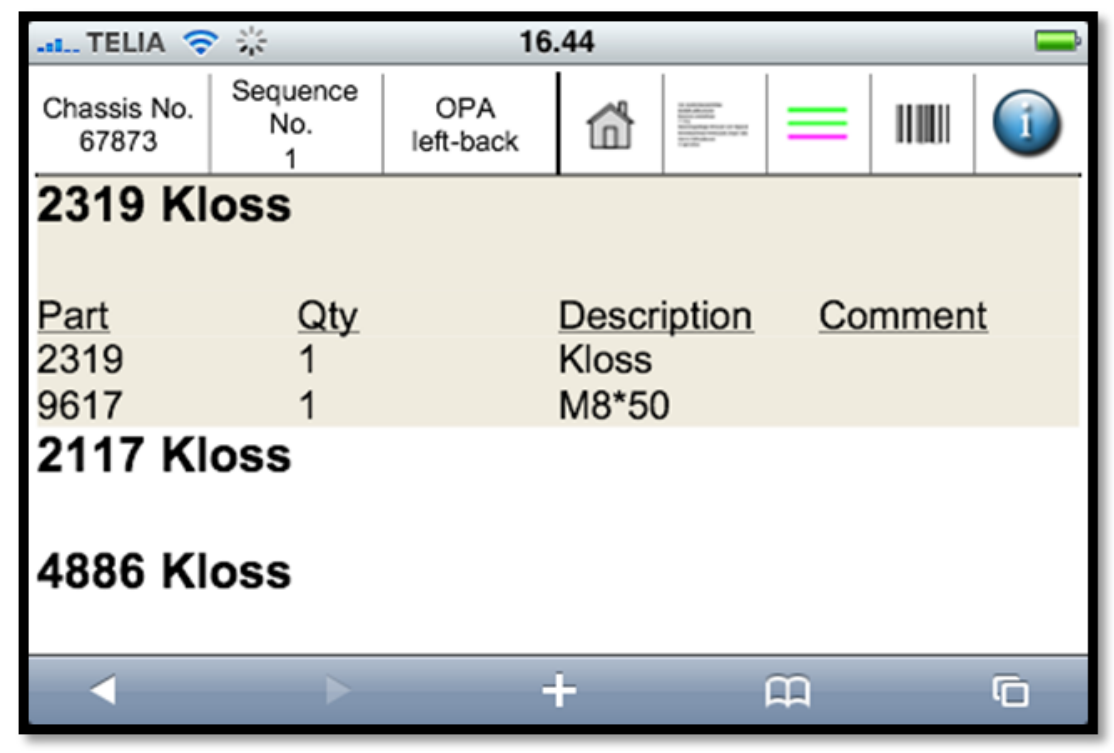

Figure 3. The interface of the software as seen in the PDA. The screen is tough sensitive and a tap on each subtask headline shows its contained parts.

The test consisted of, as mentioned earlier, two independent groups performing the same task which is assembling building blocks to a wooden chassis. One group used a computer terminal as information conveyer and the other used the PDA. The dependant or measured variables were:

1. Productivity - total time.

2. Quality - number of errors. Wrong blocks or wrong bolts.

As the subject worked on one side, a researcher disassembled blocks from the chassis on the other side.

There is an inherent risk in all types of tests where researchers are involved in operations, in this case disassembling parts during the test, that they may disturb or somehow bias the results. However, this is, first of all, balanced between the groups as both conditions have the same possible disturbance from the researcher. Secondly, these kinds of disturbances, and even worse, are expected in the actual assembly domains that we are trying to emulate. Thus there should be no confounding of the data and this fact merely adds to the external validity of the test. 


\section{RESULTS}

A total of 26 subjects took part in the experiment with 12 subjects performing the tasks with a computer and 14 subjects using the PDA as information source. The groups were evenly balanced with respects to age and gender.

For productivity the groups differed in total time with the means 41,9 (Computer) and 39,5 (PDA) minutes, as can be seen in table 1. Analysis between the groups with respect to time proved not significant in a 2-tailed independent samples t-test $(p=0,134)$. Effect size was 0,59 and post hoc statistical power 0,579 (Cohen, 1992).

Table 1. Descriptive statistics of productivity measured in minutes.

\begin{tabular}{|ll|r|r|r|r|}
\hline \multicolumn{2}{|c|}{ Group } & $\mathrm{N}$ & \multicolumn{1}{c|}{ Mean } & Std. Deviation & Std. Error Mean \\
\hline \multirow{2}{*}{ Total } & Computer & 12 & 41,8750 & 2,96284 &, 85530 \\
& PDA & 14 & 39,4643 & 4,62628 & 1,23643 \\
\hline
\end{tabular}

The mean number of errors for both groups was 3,36 as can be seen in the descriptive statistics in table 2. The participants in the computer group made an average of 4,58 errors while the participants in the PDA group made an average of 2,14 errors. For analysis of this dependent variable a non-parametric test was chosen as the distributions are slightly skewed. A 2-tailed Mann-Whitney test showed significant difference between the groups $(p=0,045)$.

Table 2. Descriptive statistics of quality measured in number of errors.

\begin{tabular}{|rl|r|r|r|r|}
\hline & Group & N & \multicolumn{1}{|c|}{ Mean } & Std. Deviation & Std. Error Mean \\
\hline \multirow{2}{*}{ Quality } & Computer & 12 & 4,58 & 3,397 &, 981 \\
& PDA & 14 & 2,14 & 2,143 &, 573 \\
\hline
\end{tabular}

Notable for the analysis of quality is that the effect size was large (ES=0,81). This is probably the main reason why a significant difference between the groups could be shown with only 26 participants in the test. A histogram for both groups showing the difference in distributions can be seen in figure 4 . The figure illustrates both differences between means and also the differences in width of distributions which is also evident through the major differences in standard deviations. Although, 
homogeneity of variances tests did show that variances between the groups were equal.

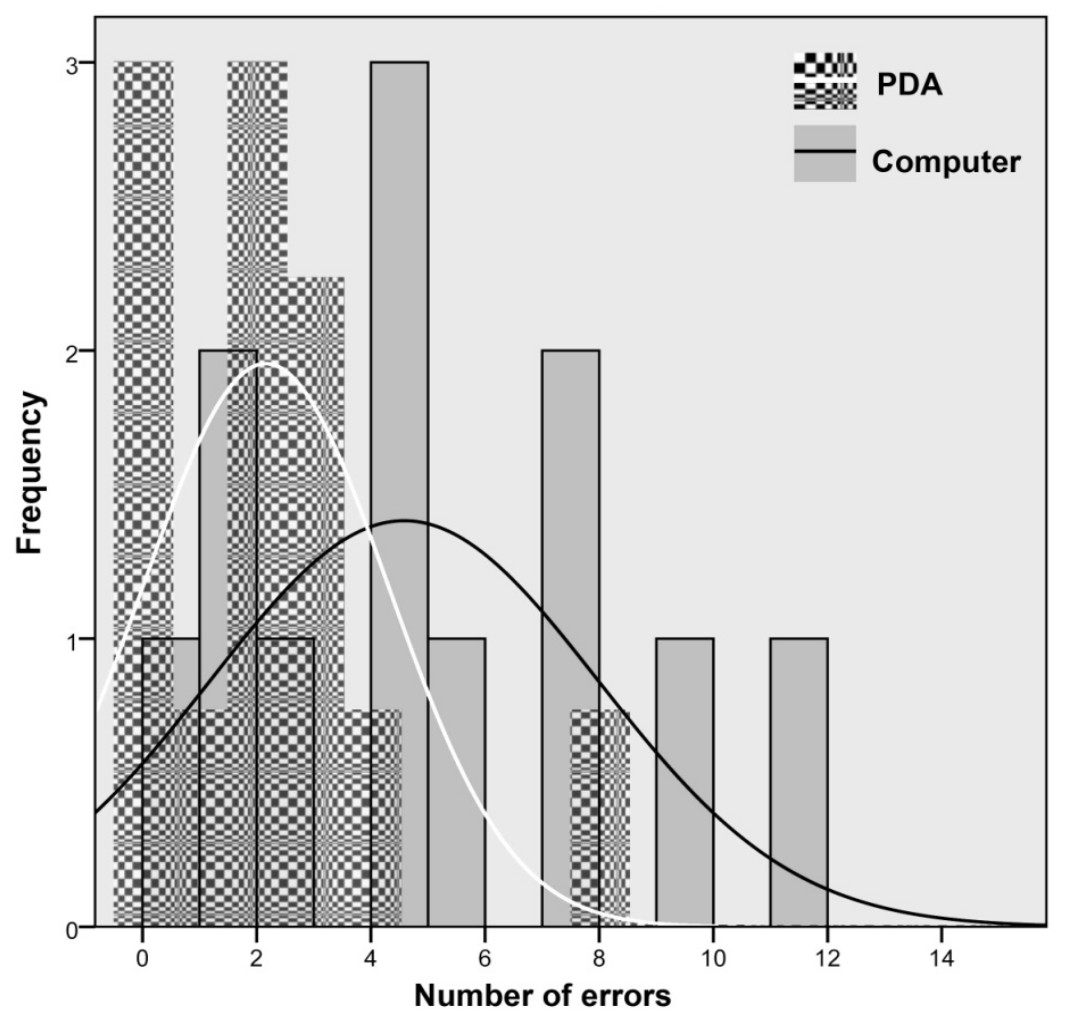

Figure 4. A histogram showing the distribution curves of the two groups.

\section{CONCLUSIONS}

Although productivity did not show a significant difference in our analysis this is thought to be because of the poor statistical power of the experiment (Cohen, 1992). The focus in the experimental design was to design quality hazards and avoid floor effects in number of errors. Post hoc statistical power for productivity was 0,579 , which gives us about a $57 \%$ chance of finding a significant difference, should there be one. More subjects would probably have resulted in this variable also being significant. Also, the reinforced hypothesis that subjects using the PDA are more inclined to use the information source probably results in their measurement for productivity being increased. The time spent on the information source obviously affects productivity if productivity is seen as an isolated factor. Naturally, in 
production, productivity is also affected by quality from an organizational point of view.

However, quality did show significant differences between the groups. This reinforces the hypothesis and the argument behind it that subjects are more prone to use the information source if it is more accessible to them. This is thought to include both physical effort and time wasted to gather this information.

To conclude, the experiment has shown how quality is greatly improved by a mobile information source. Differences in the means show that the group using the PDA produced less than half the number of errors compared to the computer-group. However generalization and applications to industry of these results must be made with great care. The experimental design was made to generate errors and these built in "traps" might not correspond fully with state of the art industry. However, many of these errors have been observed in modern industry and are a compilation of errors found through personal experience as an assembly worker and through observation.

To sum up, these results are valid for application in industry if handled with care. Since the experiment has been designed to generate as many errors as possible and the task might be considered trivial, analysis within groups may be somewhat distorted. However, the difference between groups, which is what is being investigated, is there and is significant. The main result: 'mobile information

sources result in better quality than stationary ones,' is valid and confirms previous, unpublished, observational studies.

\section{ACKNOWLEDGEMENTS}

This work has been made possible with great help from the EU integrated project MyCar. Many thanks also go to the subjects who participated in the study.

\section{REFERENCES}

Bäckstrand, G., de Vin, L. J., Högberg, D. \& Case, K. (2005). Parameters affecting quality in manual assembly of engines. In Proceedings of the International Manufacturing Conference, IMC 22, Institute of Technology, Tallaght, Dublin, August. pp. 165-172. 
Cohen, J. (1992). Statistical power analysis. Current Directions in Psychological Science, 98-101.

Dix, A., Ramduny, D. \& Wilkinson, J. (1998). Interaction in the large. Interacting with Computers, 11 (1), 9-32.

Jonides, J. \& Mack, R. (1984). On the cost and benefit of cost and benefit. Psychological Bulletin, 96 (1), 29-44.

Thorvald, P., Bäckstrand, G., Högberg, D., de Vin, L. J. \& Case, K. (2008a). Demands on Technology from a Human Automatism Perspective in Manual Assembly. In Proceedings of FAIM2008 Skövde, Sweden, June-July 2008. pp. 632-638.

Thorvald, P., Bäckstrand, G., Högberg, D., de Vin, L. J. \& Case, K. (2008b). Information Presentation in Manual Assembly - A Cognitive Ergonomics Analysis. In Proceedings of NES2008, Reykjavik, Iceland, August, 2008.

Wigfield, A. \& Eccles, J. S. (2000). Expectancy-Value Theory of Achievement Motivation. Contemporary Educational Psychology, 25 (1), 68-81. 